\title{
Sexual rights, mental disorder and intellectual disability: practical implications for policy makers and practitioners
}

\author{
Helen Dewson, Keith J. B. Rix, Isabelle Le Gallez \& Kartina A. Choong
}

Helen Dewson has an LLM in Mental Health Law from Northumbria University and currently works as a legal practice advisor for a mental health trust. She also sits as a specialist lay member and CAMHS specialist member of the Mental Health Tribunal. Keith Rix is an honorary consultant forensic psychiatrist,

Norfolk and Suffolk NHS Foundation Trust, and Visiting Professor of

Medical Jurisprudence, University of Chester, where he is involved with its MSc in Medicolegal Practice. He is an elected Honorary Fellow of the Faculty of Forensic and Legal Medicine of the Royal College of Physicians. Isabelle Le Gallez is due to complete her LLM in Medical Law and Ethics at the University of Edinburgh, having graduated from the University of Sussex with an LLB in Law, and she is about to commence a $\mathrm{PhD}$. Kartina A. Choong is a reader in Medical Law and Ethics at the University of Central Lancashire. She is a non-practising barrister and an accredited mediator. She is a Fellow of the Higher Education Academy. Correspondence Helen Dewson, Legal Services, Endeavour House, Ipswich IP1 2BX, UK. Email: helendewson@gmail.com

\section{Copyright and usage}

(C) The Royal College of Psychiatrists 2018

\section{SUMMARY}

Clear policies regarding sexual expression, sexual behaviour and related decision-making assist in ensuring that the rights of people with mental disorder or intellectual disability are upheld, and that staff know how to react to situations consistently and lawfully without interfering on the basis of their own moral judgements or personal beliefs. Sensitive and holistic planning of care that complies with domestic law, international human rights law and statutory guidance is necessary to complement such policies. Non-intimate physical contact, masturbation, sexual relationships, contraception, sterilisation and vasectomy, pregnancy, termination of pregnancy, sexual dysfunction, parenthood, marriage and civil partnership, divorce, prostitution, pornography, and sex aids and toys are all matters that may properly be part of care planning.

\section{LEARNING OBJECTIVES}

- Understand the limited legal basis for the formulation of policies and rules concerning sexual expression, sexual behaviour and related decision-making by people with mental disorder or intellectual disability

- Be able to formulate policies concerning sexual matters as they relate to people with mental disorder or intellectual disability

- Be able to plan care for psychiatric patients and community service users balancing their rights to sexual fulfilment with the protection of their own welfare and the protection of others

\section{DECLARATION OF INTEREST}

None.

\section{Keywords}

Intellectual disability; service users; in-patient treatment; sexual rights.

'The UK appeared the most prohibiting and excluding, its protocols apparently based on risk aversion and lack of emphasis or consideration of patients' sexual needs' (Tiwana 2016).

In our previous article (Le Gallez 2018) we set out the law relating to sexual expression, sexual behaviour and related decision-making on the part of people with mental disorder or intellectual disability. In this second article, we explain why policies are necessary in this area of mental health and community care, we set out their limited legal basis and we consider a range of issues that may need to be addressed in policies and individual care plans.

\section{A place for policies}

In Re SS [2001] Mr Justice Wall criticised the psychiatric hospital in which a pregnant woman with schizophrenia, SS, was detained and said that 'each hospital should have a protocol to deal with possible terminations of pregnancy in good time $[\ldots]$ so that wherever practicable [...] a termination can be carried out at the earliest opportunity'. This is only one issue relating to the sexual rights and related behaviour of people with mental disorder or intellectual disability for which a protocol or policy is desirable, if not necessary.

The Royal College of Psychiatrists (1996) had already recommended:

'Each unit should have a clear written policy which covers acceptable, consenting activity and issues such as harassment and sexual abuse. The policy should ensure that sexuality and sexual issues are considered as part of individual care plans.'

Craft (1987) has identified the advantages of policy guidelines for people with intellectual disability (Box 1). Those who provide treatment or care for people with mental disorder or intellectual disability have commensurate obligations to ensure that the delivery of care does not breach legislation. Service providers are responsible for ensuring that staff have the necessary up-to-date knowledge and skills to identify potential issues or difficulties. They are obliged to provide consistently lawful, ethical and 
equal care to all psychiatric patients and community service users. Policies and guidance should be available to support identifying, learning, reporting and escalation, so as to avoid placing staff in situations that they feel inadequately informed to address or where they may make ill-informed decisions. Service providers will be liable for unlawful acts committed by their employees unless they are able to demonstrate that reasonable steps have been taken to prevent such acts (s.109 of the Equality Act 2010).

As observed by Bartlett et al (2010), 'the absence of clear policy increases the probability that staff will be guided by their own moral judgements and personal beliefs, and hence act inconsistently as a group'.

Policies are also important because there is an 'ongoing tension between helping people to realise their right to develop or maintain relationships, and engage in sexual relationships should they wish to do so, and the need to protect vulnerable people from abuse and exploitation' (Cheshire County Council 2015: p. 4 ('the Cheshire policy')). It is a complicated area of practice. The person who wants to develop a relationship may seek to form it with someone who is vulnerable, but the person seeking to form the relationship may be vulnerable to abuse or exploitation too (Fyson 2015).

\section{Policies and the law}

In any institution, or in any service in the community, where people live or come together, there need to be sensible rules to ensure smooth running, the protection of interests and rights, maintenance of standards of common decency, and the avoidance of behaviour that upsets or offends individuals or disturbs the community or the group. However, there is a limited legal basis for making the rules that policies embody. Those who formulate policies need to be aware of this and be informed by relevant legislation, case law and statutory guidance (see Le Gallez 2018).

Bartlett et al (2010) are of the view that for the sort of intrusive, and in some cases extraordinarily intrusive, rules in this area, they would expect a clear legal rule-making authority, based in statute and, perhaps, secondary legislation. They observe that, in contrast to the prison context, such a structure is conspicuously absent in the context of psychiatric facilities.

For patients detained under the Mental Health Act 1983, the court recognised in $R v$ Broadmoor Special Hospital Authority (1998) that 'the express power of detention must carry with it a power of control and discipline' including, for example, 'the power [...] to regulate the frequency and manner of visits to [patients]'. However, it is difficult to see that this is a legal basis for 'rules of
BOX 1 Advantages of policy guidelines relating to the sexual expression of people with an intellectual disability
Advantages for the individuals

Explicit acknowledgment of their right to be sexual beings/to grow up/to have ordinary life experiences

Safeguard against the idiosyncratic beliefs, attitudes and responses of individual staff members

A more enabling/empowering environment and atmosphere

Relationships are valued rather than belittled

Privacy is respected

Appropriate sociosexual education as a matter of course rather than prompted by a crisis Availability of a counselling service for specific needs

Dignity and respect enhanced

\section{Advantages for staff}

Boundaries of acceptable behaviour become clear/defined

Managers have to give realistic and constructive consideration to the issues and to place the statements on record

Legal position clarified

Responsibility for teaching/counselling clearly allocated

Problematic areas can be openly discussed within an enabling structure

Counselling service for staff

Management decisions are facilitated and become less anxiety-provoking

Gives a baseline in discussions with patients or service users

Training needs are acknowledged and provided for

Involvement in the process strengthens commitment to the policy and is in itself a valuable experience governance not directly related to detention, into which category the policies on sexual and emotional expression arguably fall' (Bartlett 2010). Furthermore, in this case, relying on $R v$ Home Secretary, ex p. Leech [1994], the Court endorsed the rigorous test that for a power for which a policy contended, there had to be 'a self-evident and pressing need'. Those who formulate policies must ensure that they pass this rigorous test.

The approach of the courts to smoking in hospital is of relevance to policy formulation, particularly 'blanket bans'. In the Rampton Hospital case concerning the legality of its smoking ban $(R(N) \mathrm{V}$ Secretary of State for Health [2009]), relying on Kay $v$ Lambeth LBC [2006], the court held that 'subject to duties owed to patients or staff, it can set the rules'. However, as Kay was a housing case, where tenants could leave if they did not like the rules, its application could, as Bartlett et al (2010) observe, result in 'a marked disproportion of power in setting institutional rules'.

$R(N)$ was cited in McCann $v$ State Hospitals Board for Scotland [2017]. The hospital had introduced a blanket ban on smoking. The Supreme Court determined that a comprehensive ban on smoking was within the ambit of Article 8 of the European Convention on Human Rights (ECHR), 
in that it interferes with a patient's right to respect for his private life. Although this ban was justified as a proportionate measure because of the risks of secondary smoke under Article 8(2), the ruling marks a shift in favour of personal autonomy and respect for an individual's rights and freedoms.

There is the related issue of the individual's right to do to themselves what is physically or morally harmful or dangerous. As established in Pretty $v$ United Kingdom (2002), for those individuals with capacity to decide, 'the ability to conduct one's life in a manner of one's own choosing may also include the opportunity to pursue activities perceived to be of a physically or morally harmful or dangerous nature for the individual concerned'. The autonomous right of a capacitous adult to determine what shall be done to his or her body is a fundamental civil right, recognised and protected by law.

For those who lack capacity, the Mental Capacity Act 2005 (the MCA) provides that any act done, or decision made, on their behalf must be in their best interests. Best interests decisions must include consultation with family, and consideration of previously expressed wishes and behaviour. This may include previous sexual relationships and behaviour.

The Care Quality Commission (2017) has produced guidance on the use of 'blanket restrictions' It makes reference to the Mental Health Act Code of Practice (Department of Health 2015), which states,

\begin{abstract}
'Blanket restrictions should be avoided unless they can be justified as necessary and proportionate responses to risks identified for particular individuals' (para. 8.5)

'Blanket restrictions should never be for the convenience of the provider. Any such restrictions, should be agreed by hospital managers, be documented with the reasons for such restrictions clearly described and subject to governance procedures that exist in the relevant organisation' (para. 1.6).
\end{abstract}

BOX 2 Some positive statements for incorporation in a policy relating to sexual expression

- Everyone has the right to be treated as an autonomous individual and to be treated with respect and dignity at all times

- Each person has a right to express their sexual feelings and develop consensual relationships of their own choosing without condemnation or censure, but subject to consideration of the rights and feelings of other patients and staff and within the limits of the law

- Each person has a right to be informed and educated about sexual matters and to receive information that is meaningful and appropriate for their needs and sufficient for them to make informed choices about sexual expression, relationships and behaviour

- Patients and service users have the same rights as any citizen to receive advice and support, including: sexual and relationship counselling; marriage guidance; family planning; advice regarding abortion, sterilisation and sexual difficulties; and sexual health and well-being services

- Each person has a right not to be exploited or abused
It follows that for blanket rules created by policies, there has to be a self-evident and pressing need, such as ensuring the safety of patients or preventing harm; and for any interference with rights or freedoms to be lawful, it must be qualified and proportionate.

\section{General principles}

\section{Situation or location specific}

Policies should be tailor-made to each specific location or situation. No one policy can address all of the circumstances in which people with mental disorder or intellectual disability seek to express their sexual desires or make related decisions (Siebers 2012). A policy for a group home for intellectually disabled people of both genders will not be the same as the policy for patients housed in single-gender wards in a high secure hospital.

\section{Consultation}

The first step is to consult those who will be affected by the policy, including, but not necessarily limited to, patients or service users, carers, family members, and staff and partner organisations such as local authority social services departments. Consultation with people who have an intellectual disability should make allowances for their possible difficulties in understanding information and questions and in articulating their responses.

\section{Positive not negative}

Policies should begin with a number of positive statements (Box 2). Which of these are incorporated, and how, will depend on the circumstances. Policies should set out the responsibilities that go with the rights, such as respecting the rights of others to freedom and choice, and respecting the sensitivities of staff.

\section{Staff support and staff responsibilities}

Policies should provide for staff support, addressing the sensitivities and concerns of staff whose personal value system, culture or religion makes it difficult or impossible for them to come to terms with particular patient behaviours, for example same-sex relationships. Although staff must not allow their own cultural, moral or religious beliefs to conflict with the rights to sexual expression and the development of sexual relationships of their patients or service users, managers and supervisors must recognise the difficulties that some staff may have and support them non-judgementally and with respect and sensitivity (Box 3). As stated in the Cheshire policy 'there should be no expectation that a staff member should be required to change their own 
cultural, ethical, moral or religious codes in order to support a service user' (Cheshire County Council 2015: p. 8).

Balanced against this, however, is the equality duty placed on all public sector employees by the Equality Act 2010 to ensure that, while in the course of their employment, staff adhere to their legal requirements. Individual employees may be held personally liable under the Equality Act for unlawful acts that they commit in the course of employment, and the employer will be liable for unlawful acts committed by their staff unless they have taken reasonable steps to prevent such acts (s.109, Equality Act 2010).

\section{Training}

A cross-sectional survey of 650 mental health workers working in London found that most reported not engaging in sexual health promotion activities (Hughes 2009). Not doing anything to enable patients and service users to achieve sexual well-being can be as much a violation of their rights as deliberately curtailing such rights.

For various reasons many mental health professionals find it difficult to start a conversation about sexual matters with patients or service users. Sometimes it is because they do not want to be perceived as making sexual advances. Sometimes patients are sexually forward and the reaction of staff is to retreat behind a ban on sexual activity as a way out when, handled differently, it could be a health promotion opportunity or an opportunity for a positive conversation about sexuality. Staff often report struggling to set boundaries with patients and admit to difficulties in de-escalating the situation when patients are being sexually aggressive or inappropriate. There is therefore a need to provide staff with training in how to start and handle positive conversations about sexual and related matters and how to stop such conversations where the effect may be negative.

\section{Capacity}

As capacity is a preliminary and fundamental consideration in many of the difficult cases for which policies will have to make provision, policies need to draw attention to the relevant parts of the MCA. Whether or not the person has a mental disorder that impedes choice as to sexual activity is relevant in relation to the Sexual Offences Act 2003.

There is a need to ensure consistency with, and make appropriate cross-reference to, other policies, such as safeguarding, information sharing, confidentiality, equality and diversity. All policies need to comply with the Equality Act 2010 and, in relation to information sharing and confidentiality, the

\section{BOX 3 Staff attitudes}

$M$ is a patient in a rehabilitation ward. She is in a same-sex marriage with $P$. There are no concerns about her capacity in relation to her marriage and physical contact with her wife. She is permitted to demonstrate the same level of physical affection with her wife as other patients are permitted in the open lounge area. In the communal area they hold hands, they hug and show each other affection, they call each other pet names and generally behave in a loving affectionate way. In ward reviews, M prefers to have her wife sit alongside her, holding her hand and generally supporting her. Two members of staff find this situation difficult. Staff member A holds no religious views but is generally homophobic. Staff member B believes that same-sex marriage conflicts with her religious beliefs. Both members of staff have undertaken their required equality training.

Staff member A refuses to make eye contact with patient $M$, refuses to acknowledge M's wife as next of kin, and tells them that their behaviour is a bad influence on the younger patients.

Staff member $B$ is kind and considerate to $\mathrm{M}$, and explains that her religious views make it

General Data Protection Regulation and the Data Protection Act 2018.

\section{Boundaries}

Staff boundaries should be made clear and reference made to offences under the Sexual Offences Act 2003, which prohibits any sexual activity ${ }^{\mathrm{a}}$ between a care worker and a person with a mental disorder while that relationship of care continues. Such behaviour includes acts, words or behaviour designed or intended to gratify sexual impulses or desires (for more detail see Le Gallez 2018). Staff who ignore this prohibition risk disciplinary action as well as criminal proceedings. The care worker, even in the absence of objection from the patient, would also be liable to prosecution under s.44 of the MCA, which prohibits the ill-treatment of those who lack capacity. This is insofar as a lack of consent for sexual relations could be interpreted as ill-treatment.

\section{Diversity}

Diversity must be recognised and respected, and equality under the law upheld. Policies must not be discriminatory on the basis of age, marriage and civil partnership, pregnancy and maternity, a The Sexual Offences Act 2003 states that: 'penetration, touching or any other activity is sexual if a reasonable person would consider that -

(a) whatever its circumstances or any person's purpose in relation to it, it is because of its nature sexual, or

(b) because of its nature it may be sexual and because of its circumstances or the purpose of any person in relation to it (or both) it is sexual'. 
race, religion or belief, gender, gender reassignment or sexual orientation. It would be discriminatory to have different rules pertaining to homosexual/heterosexual behaviour or married/unmarried patients, or for those of different genders, unless qualified. For example, it is discriminatory to allow spouses of different genders private access to the patient's room while disallowing spouses of the same gender the same level of privacy.

\section{Review}

As with any policy, it should be regularly reviewed.

\section{From policy to care planning}

In many instances, a policy will provide guidance of a general nature but not be sufficient to identify and guide all the actions to be taken in an individual case. So, it is highly important that sexuality and sexual issues are considered as part of individual care plans. In the remainder of this article, we will discuss how sexuality and sexual issues can be accommodated in care plans and give some examples. A clear plan of escalation should be identified to support staff in such circumstances.

\section{The right not to have sexuality and sexual issues in one's care plan}

Although some people with mental disorder or intellectual disability welcome and accept care that

\section{BOX 4 Capacity and best interests}

Patient $X$ has a mild intellectual disability. He was admitted to hospital initially with depression. He has progressed well and is currently having trial overnight home leave. He has a long-term girlfriend, Y. They live together in a supported living facility. He has previously been assessed as lacking capacity in relation to finances and decisions about where to live. Nursing staff are concerned about $X$ re-establishing a sexual relationship with $Y$ during home leave.

The consultant and the nursing team conduct a capacity assessment in relation to his expectations of resuming sexual relations with $Y$ during leave. They are all satisfied that he is able to understand his own feelings and wishes, and those of his girlfriend, and his responsibilities towards himself and towards her.

Staff at the supported living home also discuss the issues with $Y$. They determine that she does not understand all the issues in relation to resuming a sexual relationship. However, she clearly states that she loves
$X$ and that she is happy with the physical intimacy they have previously enjoyed. The details of their physical relationship are explored through pictures. A best interests decision is made that the relationship $X$ and $Y$ enjoy is important to $Y$, and that she derives a great deal of happiness from it. The decision is made that it is not in her best interests to interfere with the physical side of the relationship. A similar process is conducted in relation to contraception, and it is determined by all that it is in her best interests to receive a depot contraceptive to avoid an unwanted pregnancy. A care plan is devised to include close monitoring of the wishes and feelings of both $X$ and $Y$ in relation to sexual contact, and their views on pregnancy.

Some months later, $X$ and $Y$ decide they would like a child. It is determined that there is no legal authority to interfere with this right, and the depot contraception is stopped. The care plan reflects the need for immediate referral to relevant services in the event a pregnancy is established. addresses their sexuality or sexual needs, for some people this is such an intensely private aspect of their lives that they do not want even to discuss such matters with those providing their care. No one should be forced to have their sexuality or sexual issues addressed in a care plan unless it is relevant, proportionate and necessary.

\section{The right time to address the issues}

Care must be taken to choose the right time to enquire as to a patient's or service user's sexuality and sexual needs. The issues may best be addressed once the person has settled on the ward or in the service, and by the team member who has the best rapport with them.

\section{Person-centred care}

What a patient or service user who has the requisite capacity is allowed to do in terms of sexual behaviour or relationships should not ordinarily be discussed or decided by staff in their absence and then, as it were, a prescription, or more likely a proscription, issued. The process should be person-centred, with the patient or service user engaged in a dialogue from the beginning and involved in the decision-making and planning throughout. This best ensures that the care is tailored to the individual's needs and not based on assumptions by staff as to what should be done or, worse, that it does not reflect the personal beliefs and moral judgements of staff. Also, there is certainly no place for printing a standard care plan for sexual expression and filing it with the other care plans. Account needs to be taken of the life experiences, circumstances, beliefs and values that make the person unique. Although the MCA begins with the principle that a person must be assumed to have capacity unless it is proved that they lack it, staff need to ensure that specific capacity assessments are undertaken where appropriate, followed by any necessary best interests decision meetings (Box 4).

\section{Family and carers}

Although often someone's sexual life will be something they keep private from family, friends or carers, in some circumstances it may be appropriate, subject to consent being given, for others to be involved. Most obviously there will need to be involvement of the spouse or partner when plans are being made that have a bearing on their relationship. Where a young intellectually disabled adult is contemplating marriage, they may be assisted by discussion with their parents or carers. But, particularly with people who are intellectually disabled, staff need to guard against 
parents or carers, albeit well-meaning, who are protectively minded or cautious. Some parents or carers may not want their offspring or charges to receive private sex education or contraception, as happened in Gillick v Wisbech and West Norfolk Health Authority [1986]. The concerns of family members and carers should be addressed, but the overriding consideration will be the best interests of the patient or service user.

\section{Record-keeping}

Records relating to the sexuality, sexual needs or sexual behaviour of a patient or service user must be made confidentially, with sensitivity and non-judgementally. Lurid, sensational or inappropriately explicit form or content should be avoided. No more should be recorded than is necessary.

\section{Collaborative care planning}

Where a patient or service user is in, or contemplating, a relationship with another patient or service user, it may be necessary not only for issues to be addressed in both care plans, but also for the two different or overlapping teams to meet and discuss the cases jointly. However, the confidentiality of both parties must be maintained and information only shared either with informed consent or in the best interests of one or both parties if one or both parties lack the capacity to consent.

\section{Reporting to the police and safeguarding authorities}

Within the context of protecting and empowering individuals, safeguarding considerations apply to both the individual and to others who may be at risk of harm or exploitation. A thorough safeguarding identification and reporting system must be in place, along with policies relating to when safeguarding referrals to the local authority are appropriate to enable a multiagency safeguarding enquiry to be implemented under the Care Act 2014. Such enquiries promote outcomes that both protect and support independent choices while protecting individuals from harm.

\section{Specific issues in policy-making and care planning}

The following issues may call for the formulation of policies and need to be addressed in individualised care plans.

\section{Non-intimate physical contact}

As the title of the paper by Bartlett et al (2010) indicates - 'You may kiss the bride, but you may not open your mouth when you do so' - policy writers may be tempted to draw a line and separate acceptable non-intimate affectionate physical contact from non-acceptable sexual behaviour. It is a moot point whether kissing with the mouth closed is sexual behaviour. That it is a moot point illustrates both the need for careful definition and the difficulties that may arise when such strict definitions are applied.

It is common to see references in medical records to 'inappropriate sexual behaviour'. However, sometimes enquiry reveals that the inappropriateness is a subjective interpretation based on many individual factors, and it is behaviour that in all circumstances other than, for example, a psychiatric ward or a day centre, would be regarded as appropriate; and there may not be unanimity among the staff as to its inappropriateness. As Rosen observed almost 50 years ago, 'institutions are generally not good at helping people distinguish between acceptable and unacceptable sexual expression' (Rosen 1972, cited by Craft 1987) and it is the experience of two of us (H. D. and K. R.) that this remains so. Flirting is often cited as 'inappropriate sexual behaviour' even in instances where it does not cause offence or concern. It should be clear why such behaviour is inappropriate and has been added to a patient's risk profile. 'Flirting' by a convicted sex offender with a history of grooming may well be a risk behaviour and appropriately flagged. 'Flirting' between individuals without such a risk profile will need to be evaluated within the context of normal human behaviour. 'Sexting' using mobile phones or computers may require additional consideration in terms of risk assessment and monitoring.

If distinctions are to be made between inappropriate/unacceptable and appropriate/acceptable behaviour or if, for example, kissing or hugging are permitted in some areas but not others, clear definitions and reasoning must be provided.

\section{Masturbation}

Masturbation is a normal sexual activity for many people. Policies must include respect for patients' and service users' right to masturbate in private without interference, censure or condemnation by those who care for them. Policies may also need to make it clear that masturbation in the presence of others, unless it is with their consent and in private, is unacceptable. Where an individual wishes to purchase masturbatory aids such as vibrators, passive or overt criticism avoided and any invasive questioning should be qualified. 
BOX 5 Intellectual disability and sexual relations in marriage: the case of $\mathrm{CH}$

CH was 38 years old. He has Down syndrome and an associated intellectual disability. He had been married for 4 years to WH. She does not have a mental disorder or intellectual disability. Since they married, they had been living together in $\mathrm{CH}^{\prime}$ 's parents' home. $\mathrm{CH}$ and WH enjoyed normal conjugal relations. The nature of their relationship became apparent when they sought fertility treatment.

This prompted an assessment by a psychologist, who concluded that $\mathrm{CH}$ lacked the capacity to consent to sexual relationships. ${ }^{\text {a }}$ Following this assessment, the couple were informed of this conclusion and WH was advised that she must abstain from sexual intercourse with $\mathrm{CH}$ as that would, given $\mathrm{CH}$ 's lack of capacity to consent, amount to a criminal offence under the Sexual Offences Act 2003. WH was advised that if she did not comply, safeguarding measures would be taken and as a result, she or $\mathrm{CH}$ would have to be removed from the home. WH moved into a separate bedroom. CH could not understand why this had happened. WH reduced physical expressions of affection so as not to 'lead him on'.

A plan was made for $\mathrm{CH}$ to undergo a course of sex education and this was delivered by a therapist. The course was completed in the expected time frame. The therapist reported that $\mathrm{CH}$ had made sufficient progress except that he could not understand the health risks associated with sexually transmitted disease. However, this was not considered to be 'relevant information' within the meaning of the Mental Capacity Act because the couple were in a monogamous and exclusive relationship. Nevertheless, for other reasons, further sex education was advised. Following the completion of this additional course, reassessment established that $\mathrm{CH}$ now had the capacity to consent to sexual relations. $\mathrm{CH}$ and $\mathrm{WH}$ then resumed their conjugal relationship.

a. See Le Gallez et al (2018) for the law on capacity to consent (CH v A Metropolitan Council [2017]) to sexual relations.

\section{Sexual relationships}

In a study of psychiatric units in West London, Warner et al (2004) concluded, 'The amount of sexual activity disclosed by the survey was remarkably high'. Of 27 staff members who completed questionnaires, 26 believed sexual activity to be occurring in the psychiatric unit; 13 thought it happened often.

The starting point should be a consideration of how patients or service users can be enabled to exercise their right to maintain or form sexual relationships in the particular institution or service to which the policy applies.

\section{BOX 6 Where is private?}

'Sexual expression is essentially a private matter, but where is private? Staff have a major part to play here, because privacy has to be somewhat artificially created and then respected. So, for example, in a shared room or dormitory a person's bed may be deemed a private area; or where a couple have taken the trouble to find a quiet room away from others, that privacy should be respected. All this can only work satisfactorily within the
Issues may also arise involving people who are not in receipt of services. Box 5, a case that came to the Court of Protection, illustrates how the care planning of sex education enabled a married man with an intellectual disability, who lacked capacity to consent to sexual relationships, to resume a conjugal relationship.

In residential facilities such as care homes, shared houses, group homes, hostels or where other supported living arrangements exist, it should be recognised that residents may wish to engage in sexual activities with another person in the privacy of their own room. In other settings, this may be more challenging for staff (Box 6). Residents should be able to lock their bedroom doors, unless this is prohibited as part of their general risk profile. Staff should not, other than in exceptional and defined circumstances, enter residents' rooms without their consent unless this is as part of an existing observational level, in which case appropriate discretion and courtesy should be observed. Staff should assist, where appropriate, in making the resident's room a comfortable one in which friends can be received in private. Box 7 illustrates a problem that can arise where a married person is resident in a care home.

The corollary of this is that, under common law, it is an offence to outrage public decency by engaging in a sexual act in public. The public element is satisfied if at least two persons are present or may reasonably be believed to observe such behaviour. But even if one person is present, who may be offended by what they witness, this may legitimately be something that a policy should discourage even though it is not unlawful. Similarly, voyeurism and exhibitionism are criminal offences under the Sexual Offences Act 2003.

It is not simply a case of distinguishing between acceptable and non-acceptable venues for sexual behaviour. A community day centre, in most if not all circumstances, will not be an acceptable venue. The ward of an in-patient unit might be if sufficient privacy exists such that no one observes, or is likely to interrupt, it. Indeed, in some facilities accommodating patients on a medium- or long-stay basis, there may be good grounds for making provision for conjugal visits to satisfy their right to a family life (Article 8(1), ECHR).

Very careful consideration needs to be given to a policy that actively discourages or bans sexual activity between patients or service users. This may infringe their Article 8 right. As the case of $R e$ MM, Local Authority X v MM and KM [2007] makes clear, 'any public body which proposes to interfere with the sexual life of someone who [...] has capacity faces a heavy burden'. 'Particularly serious reasons' must exist and, in the case of 
those who are in an ongoing relationship, and the more so if it is long-standing, 'especially pressing reasons must surely be shown to exist'.

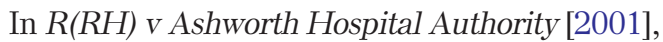
where the claimant challenged the refusal of the hospital to provide condoms for patients who were sexually active, the court accepted the hospital staff's view that a prohibition on sexual activity between patients was justified in order to manage the risks of sexual offending and sexual abuse. However, Bartlett et al (2010) have referred to the evidence given by the hospital staff in support of this policy as 'statistical nonsense' and 'not particularly convincing'. They have pointed to the inconsistency between the court's acceptance that virtually no sexual activity occurred in Ashworth Hospital, and the contrary evidence of the applicant and the contrary acknowledgment in the hospital's own policy, and they conclude that it is a moot point whether the nature of the patient population warrants a blanket 'no sex' policy. They go on to observe that the case does not stand as a precedent that a blanket 'no sex' policy can be justified outside high secure hospitals where patients are less ill and less dangerous (Box 8).

Consideration also has to be given to the potentially counterproductive effects of a ban on sexual relationships between patients. 'A simply prohibitive policy [...] risks driving the relationship into secrecy, so that those involved are effectively precluded from talking about the relationship and its effects on them' (Bartlett 2010). This places outside risk management issues such as venereal infection and, if the relationship is heterosexual, pregnancy. Bartlett et al (2010) also point out how a blanket ban can work against the eventual well-being of patients who are married or in long-term relationships for whom '[u]pon release, it is of course highly desirable that the individuals have a secure social system in the community, typically provided through family relationships'. Indeed, it is arguable that for such patients, facilitation of a sexual relationship may be in the patient's, and their partner's, interests as the relationship will be under closer scrutiny than following discharge and, if it is required, immediate support or intervention can be provided.

\section{Contraception (including emergency contraception)}

Patients and service users may wish to use contraception to avoid pregnancy or to reduce the risk of contracting a sexually transmitted disease (STD). Information and advice should be available, including that provided by specialist services such as family planning services. It may be advisable to initiate such discussions where staff are aware that an

\section{BOX 7 Dementia and sexual relations in a care home}

$L$ is a 78-year-old married man with dementia who lives in a care home. His placement has been arranged through the old age psychiatry services. His care coordinator, a nurse, is contacted urgently by the care home manager. A member of staff has discovered L's wife in bed with him during one of her almost daily visits. Some of the care home staff want him transferred to a psychiatric hospital. His children have been informed. They say that their parents 'Shouldn't be doing it at their age' and they want their mother's visits restricted to the communal area of the home. It is established that the couple had been having sexual relations every $2-3$ weeks before L's admission to the care home, sometimes initiated by him and sometimes initiated by his wife. Although $\mathrm{L}$ lacks capacity to consent to sexual relations, it is agreed that there is no lawful basis for preventing him from initiating sexual relations with his wife and it is agreed that when she visits, staff will afford them privacy. In considering how this differs from $\mathrm{CH}$ above (Box 4), it is important to note that each case was considered on the specific facts and with regard to the views of those assessing capacity and risk.

\section{B0X 8 Extra-marital sexual relations in a low secure unit}

$V$ is a man with schizoaffective disorder. He has no history of sexual abuse, sexual dysfunction or abnormal sexual behaviour. He is the subject of a hospital order with restrictions that was made following his conviction for offences of arson committed during a psychotic episode. W is a woman with an emotionally unstable personality disorder who was sexually abused in childhood. She is the subject of a hospital order that was made following her conviction for a number of assaults on children. They first met in a medium secure unit and, although contact was limited, were known to have formed a romantic attachment there. $V$ was transferred to a low secure unit 9 months ago and in the meantime $\mathrm{V}$ and $\mathrm{W}$ have kept in contact by letter and telephone. $W$ has now been transferred to the same low secure unit. Staff learn from another patient that $\mathrm{V}$ and $\mathrm{W}$ are having sexual intercourse in a storeroom in the rehabilitation suite during the evening social hour

The first response of the staff is to ensure that the storeroom door is locked during the social hour. Both patients are reviewed by their care teams. W's case is referred to the local safeguarding authority. Some staff want $\mathrm{V}$ and $\mathrm{W}$ transferred back to medium security. Some staff want them restricted to their wards. The care teams responsible for $V$ and $W$ arrange for the senior occupational therapist, who knows both of them, to discuss with them their relationship and any plans for the future. $V$ and $W$ indicate that they want to get married. $W$ explains that $V$ is the first man who has treated her kindly and with respect. When the occupational therapist reports back to a joint meeting of the two care teams, there is agreement that there are no grounds for seeking to interfere with the relationship between $\mathrm{V}$ and W. Both are judged to have capacity to consent to sexual relations. It is decided that contraception and sexual health should be addressed in their care plans.

$V$ and $W$ are relieved that staff have found out about their relationship. They ask if they can be allowed into each other's room so that they can be together in private. Staff explain that male patients are not allowed in the rooms of female patients and vice versa. However, as $\mathrm{V}$ and $\mathrm{W}$ plan to live together following discharge, agreement is reached to allow them to spend an occasional night together in the unit's rehabilitation flat in between the periods of several days when it is occupied by other patients. This arrangement is incorporated into their care plans and reviewed on a regular basis. When, many months later, supported accommodation is identified for them in the community, they progress to spending several days at a time in the rehabilitation flat. Eventually they are discharged to live together. 
individual is sexually active. Policies should recognise such wishes and set out how information and advice can be sought.

For people who lack capacity to consent to contraception but possess the capacity to consent to sexual relations it may be appropriate to hold a best interests decision meeting to consider the provision of contraception. This might include sexual education and provision of condoms, or the provision of longacting depot contraception.

Where exceptionally access to contraception such as condoms is forbidden, policies need to justify such a prohibition. If the purpose of the policy is to discourage sexual activity, the policy itself may place the individual at risk of STD or pregnancy. Such an outcome is contrary to the individual's own health and safety and that of the wider public.

Policies should include consideration of emergency contraception because its effectiveness is related to how soon it is taken. Staff, service users and patients need to be aware of its availability and effectiveness. Staff should be prepared to help service users in seeking such treatment.

\section{Sterilisation and vasectomy}

Patients and service users may seek sterilisation or vasectomy. Staff should be in a position to advise, support and inform them. Particularly in the case of people with intellectual disability, family members or carers may suggest sterilisation or vasectomy. Policies need to make it clear that sterilisation and vasectomy cannot be performed on people with capacity to consent without their permission or on people who lack capacity unless a court decides that it is in their best interests. Policies must identify the steps to be taken to obtain a decision of the Court of Protection.

\section{Sexual dysfunction}

Sexual dysfunction is a common side-effect of antipsychotic medication and can be a major problem for some patients as it can affect their ability to express their sexuality and form and maintain sexual relationships. It is often not discussed with patients and service users. Not addressing such a problem can amount to an obstacle for patients in exercising their right to sexual expression.

\section{Pregnancy, termination of pregnancy and parenthood}

People with mental disorder or intellectual disability have the same rights to become parents as other citizens. When a patient with a mental disorder or a service user with an intellectual disability becomes pregnant, it may be of little or no concern to those responsible for their care beyond sharing their delight or supporting them in their distress. But policies must provide for the provision of appropriate support, education and assistance for those who are unprepared for parenthood and for those who will only make a success of parenthood with appropriate care. It may be necessary to take into account not only how the mother will cope with the child but any limitations the father may have and the help the father may also need. Early referral to the relevant services is of paramount importance.

A woman who is uncertain about continuing with her pregnancy may require prompt assistance in weighing up the advantages and disadvantages of continuation and a timely referral to a pregnancy advisory service or other appropriate impartial professional advisor so as to be able to make an informed decision. If he is involved, the baby's father may have similar needs. In recognition of the fact that, for some staff, termination of pregnancy is for religious or cultural reasons contentious, policies need to ensure that the feelings, values and attitudes of staff do not compromise care.

Any best interests decision in relation to termination of pregnancy must place the patient at the centre of the decision. The best interests of the patient override all other considerations (Abortion Act 1967). Where a patient with significant mental disability is delighted with a pregnancy, a termination may not be in her best interests because of the distress it may cause. This does not preclude subsequent best interests decisions in relation to the child in matters relating to child protection.

\section{Marriage}

The right to marry is a fundamental human right enshrined in Article 12 of the ECHR and this should be affirmed in policies. There may be little or no problem in the case of an out-patient who has the capacity to consent to marriage. It is a far more complicated matter if the person who wishes to marry is a detained patient in a high secure hospital, particularly if their intended spouse is similarly detained. It may be rare for a patient in a high secure hospital to express a wish to marry but this right is protected by law (Marriage Act 1983). It is better to have an appropriate policy than have to decide how to proceed after the issue has arisen. The MCA prohibits best interests decision makers from agreeing to a marriage where the person lacks the capacity to consent for themselves.

\section{Divorce and separation}

Policies should make it clear that patients and service users are subject to the same laws relating to divorce as other citizens. Provision needs to be made for access to appropriate advisory and 
support services, both within the service and from external agencies such as Relate, and legal advice. The MCA prohibits best interests decision makers agreeing to a divorce where the person lacks the capacity to consent for themselves.

\section{Sexual diversity}

As it is unlawful to discriminate against any person on the grounds of their actual or presumed sexual orientation when providing facilities or services, policies should draw attention to this. It may also be appropriate to spell out the need to ensure that civil partners or same-sex partners are treated in the same way as married or mixed-sex unmarried couples. In Box 9, we set out staff responsibilities in this regard from the Cheshire policy.

Box 10 illustrates an issue relating to sexual diversity.

\section{Access to prostitution}

Policies should address the circumstance in which staff become aware of a patient or service user using, or seeking to use, the services of a prostitute. Prostitution is not in itself illegal. ${ }^{\mathrm{b}}$ However, if staff are aware that patients or service users are using the services of prostitutes on their premises, this may give rise to a charge of keeping, or managing or acting to assist in the management of, a brothel. Assisting a patient or service user in obtaining the services of a prostitute could be illegal under s.53A of the Sexual Offences Act 2003.

Policies should make clear that staff who are aware that a patient or service user is obtaining, or proposing to obtain, the services of a prostitute have a responsibility to counsel the patient or service user concerning the legal, social, moral and health aspects of prostitution without being judgemental and, where appropriate, having regard to any relevant aspects of the patient's or service user's history, such as a history of violence, sex offending, victimisation or victimhood (Box 11).

It may be appropriate for policies to make it clear that staff cannot and will not condone the purchase of the sexual services of a prostitute by a patient or service user.

\section{Pornography, sex aids and sex toys}

Policies should define pornography and specify the materials covered by the policy, for example magazines, posters, films, DVDs, videos and books. A distinction should be made between pornography that it is not illegal for people over the age of 18 years to access or possess, and pornography that it is illegal to access or possess, as defined by Part 5 of the Criminal Justice and Immigration Act 2008, such as that depicting children or extreme violence.
B0X 9 Staff responsibilities with regard to sexual diversity

'Staff should:

- Be aware of different sexual orientations;

- Respect different sexual identities;

- Ensure an open and professional relationship is established which enables service users to discuss their sexual orientation if they wish to do so;

- Provide information and where appropriate support service users to access relevant community resources and specialist organisations.'

(From Cheshire County Council 2015: p. 13)

In relation to films, a distinction might be made between films classified '18' by the British Board of Film Classification, and unclassified films and 'R18' films.

Distinctions should be made between what is not permitted under any circumstances, what is freely permitted and what is permitted in certain circumstances (i.e. restricted).

Where access or possession is permitted, it should be made clear that patients or service users must recognise that some people find such materials offensive and they have a responsibility only to use such materials in private and to store them in a private place. It

\section{BOX 10 A transgender issue}

$\mathrm{G}$ is a male-to-female transgender patient in the female rehabilitation ward of a medium secure unit. She has not completed full physical transition and retains male genitalia. She sexually assaults two other female patients. The assault consists of non-penetrative groping. A request is made by the nursing staff for her transfer to a male ward. The reasons given are that she retains male genitalia and is physically strong. These factors raise the risk to other females. The senior manager, in discussion with nursing staff, agrees that it would be more appropriate for her to be nursed on the male ward as her sexual orientation is towards heterosexual females. Following case review by the consultant from the male ward, the decision is reversed on the basis that it would be direct discrimination. Instead, $\mathrm{G}$ is transferred to the acute female ward, where the staff risk assess and manage her behaviour in the same manner as they would a patient who was born female. She makes one further attempt to assault, which is managed with restraint. Thereafter, she is managed with one-to-one observations within arm's length. The behaviour reduced over a period of 2 weeks, and she was transferred back to the female rehabilitation ward. b However, in Northern Ireland it is illegal to pay for sex (Human Trafficking and Exploitation (Criminal Justice and Support for Victims) Act (Northern Ireland) 2015). 
B0X 11 Access to prostitution pital admissions, most of them under the Mental Health Act 1983. He has greatly improved and is in the process of increasing unescorted leave with a view to discharge in the next few weeks. He is allowed unescorted leave under s.17 of the Act. Staff learn, from another patient, that while on leave he has been visiting a prostitute. $R$ had been married, but the marriage was dissolved when he was 25 and he started visiting this particular prostitute when he was 30 . His risk is reassessed. He has no history of violence and, but for his belief that there is no moral objection to prostitution, he has no attitudes that might be construed as possibly harmful to women. He is aware of the health and legal risks he runs. It is pointed out to him that, although prostitution is not illegal, he is at risk of prosecution if the prostitute has been subjected to exploitative conduct. He says that he has got to know her well over the course of many years and he is sure that she has not been subjected to exploitation. Some his s.17 leave. On full consideration, the team decides, with some reservations, that there are no grounds for restricting or cancelling the leave. The team sees $R$ and it is documented that his use of the prostitute is not conbehaviour and, at their suggestion, it is put to him that there are members of society who object to prostitution on moral grounds and his acknowledgment of this is documented. A plan is put in place to monitor his sexual health.

needs to be clear, for example, that although it may be acceptable for a hospital patient to have a sexually explicit poster on the inside of a wardrobe door, it is not acceptable to have such a poster on the outside of the wardrobe door (Box 12).

\section{B0X 12 Pornography}

Thas a mild intellectual disability. He lives in a group home where his room is cleaned by a member of the domestic staff. One day the cleaner refuses to clean the room because $T$ has pornographic pictures on the wall. The cleaner says that he finds these offensive and, for religious reasons, abhorrent. T's pictures are the centrefold pages of magazines that he has bought from the local newsagent. Staff review T's history and make enquiry of him sufficiently to confirm that he has no
Since the age of $35, \mathrm{R}$ has suffered from schizophrenia. Initially it was difficult to treat and he had several hosteam members want R's responsible clinician to cancel doned. Some team members have moral objections to his

Sharing of pornography or trading it with other patients or service users should be discouraged or even prohibited, as other patients may have different risk profiles from that of the individual.

Similar consideration should be given in policies to sex aids and sex toys.

The issue of staff purchasing pornography for or on behalf of patients or service users, or otherwise obtaining or providing pornography for them, should be addressed. Staff should be advised that they should not make derogatory remarks about, or make fun of, patients or service users who use pornography or sex aids. Pornographic materials should not be used for sex education.

Employers should ensure that staff are informed by policy or guidance as to what to do if a patient or service user is found in possession of illegal pornographic material.

Consideration needs to be given to restriction of access to particular 'free to view' television channels, which may broadcast sexually explicit material. A distinction may be made between access via a communal television and access via a television in a patient's or service user's private room.

Access to social media, and forms of online sexual behaviour or online relationships, should also be subject to risk assessment and adherence to local policies. This may include the sending or receiving of sexualised images, including 'selfies'. Article 8 of the ECHR may be engaged in circumstances where a patient is prohibited from forming or maintaining relationships in this manner.

\section{Conclusions}

It is in the interests of patients, service users, their families and their carers and staff to have policies that uphold the rights of patients and service users to pursue a 'satisfying, safe and pleasurable sexual life' and to make decisions on related matters, such as sexual relationships, marriage, civil partnership, divorce, contraception, sterilisation and termination of pregnancy, with no more interference or curtailment than is relevant, proportionate and necessary having regard to domestic and human rights legislation. Such policies are the starting point for ensuring that sexuality and sexual issues are considered as part of individualised care plans. history of sexual offending or of violence towards women and has never been in a sexual relationship. A plan is set in place. T is informed about the circumstances in which some women model for such magazines. Some staff express concerns about the risk to T's morals. His right to pursue an activity that may be morally harmful to him is explained to staff. T agrees to move the pictures to the inside of his wardrobe door.

\section{Acknowledgments}

We are grateful to the Cheshire and Wirral Partnership NHS Foundation Trust for providing us with a copy of its Social, Personal \& Sexual Relationships Good Practice Guidance. Boxes 1 and 6 are taken from Craft A (ed) (1987) Mental Handicap and Sexuality. The publishers, D. J. Costello of Tunbridge Wells ceased trading in 
1996 and we have been unable to ascertain who owns the copyright.

\section{References}

Bartlett P, Mantovani N, Cratsley K, et al (2010) 'You may kiss the bride, but you may not open your mouth when you do so': policies concerning sex, marriage and relationships in English forensic psychiatric facilities. Liverpool Law Review, 31: 155-76.

Care Quality Commission (2017) Brief Guide: The Use of 'Blanket Restrictions' in Mental Health Wards. COC.

Cheshire County Council, Cheshire and Wirral Partnership NHS Foundation Trust (2015) Social, Personal \& Sexual Relationships Good Practice Guidance (CP44, issue no. 1). Cheshire County Council.

Craft A (1987) Mental handicap and sexuality: Issues for individuals with a mental handicap, their parents and professionals. In Mental Handicap and Sexuality: Issues and Perspectives (ed A Craft). DJ Costello.

Department of Health (2015) Mental Health Act 1983: Code of Practice. TSO (The Stationery Office).

Fyson R (2015) Promoting sexualities and protecting from abuse In Sexuality and Relationships in the Lives of People with Intellectual Disabilities: Standing in My Shoes (eds R Chapman, S Ledger, L Townson): 163-72. Jessica Kingsley Publishers.

Hughes E, Gray R (2009) HIV prevention for people with serious mental illness: a survey of health workers' attitudes, knowledge and practice. Journal of Clinical Nursing, 18: 591-600.

Le Gallez I, Rix KJB, Choong KA, et al (2018) Sexual rights, mental disorder and intellectual disability: principles and law. BJPsych Advances, 24: $334-45$.
Royal College of Psychiatrists (1996) Sexual Abuse and Harassment in Psychiatric Settings (Council Report CR52). Royal College of Psychiatrists.

Siebers T (2012) A sexual culture for disabled people. In Sex and Disability (eds R McRuer, A Mollows). Duke University Press.

Tiwana R, McDonald S, Völlm B (2016) Policies on sexual expression in forensic psychiatric settings in different European countries. International Journal of Mental Health Systems, 10(5): 1-11.

Warner J, Pitts N, Crawford MJ (2004) Sexual activity among patients in psychiatric hospital wards. Journal of the Royal Society of Medicine, 97 . 477-9.

\section{Law reports}

Gillick v Wisbech and West Norfolk Health Authority [1986] 1 AC 112

Kay v Lambeth LBC [2006] UKHL 10.

McCann v State Hospitals Board for Scotland [2017] UKSC 31, [2017] MHLO 22.

Pretty $v$ United Kingdom (2002) 35 EHRR 1

$R$ v Broadmoor Special Hospital Authority (1998) WL 1044171

$R v$ Home Secretary, ex p. Leech [1994] OB 198, CA

$R(N)$ v Secretary of State for Health [2009] EWCA Civ 795

$R(R H)$ v Ashworth Hospital Authority [2001] EWHC Admin 872.

Re MM, Local Authority Xv MM and KM [2007] EWHC 2003.

Re SS [2001] 1 FLR 445.
MCO answers

1 c 2 c 3 b 4 c $5 e$
MCQs

Select the single best option for each question stem

1 The legal basis for policies on sexual relations and related matters for people with mental disorder or intellectual disability:

a is now enshrined in the Care Act 2014

b was enunciated in McCann v State Hospitals Board for Scotland [2017]

c should be domestic legislation

d is set out in the Equality Act 2010

e is set out in Article 8(2) of the European Convention on Human Rights.

2 Policies concerning sexual relations and related matters for people with mental disorder or intellectual disability:

a can lawfully require staff to change their own cultural, ethical, moral or religious codes

b should have as their starting point a list of rules and proscribed behaviours

c should recognise the patient's or service user's right to pursue activities perceived to be of a physically or morally harmful or dangerous nature to the patient or service user

d can be readily formulated to apply to every location or situation in a service or institution

e are exempt from compliance with the Mental Capacity Act 2005
3 A ban on sexual relationships between patients:

a has its basis in Article 8(1) of the European Convention on Human Rights

b may be justified to manage the risks of sexual offending

c in high secure hospitals can also be readily implemented in medium and low secure psychiatric facilities

d flows naturally from the power to regulate the frequency and manner of visits to patients in high secure hospitals

e was proposed by the Royal College of Psychiatrists in 1996

4 When a patient with a mental disorder or a service user with an intellectual disability becomes pregnant:

a the Abortion Act 1967 does not apply

b the father's needs can be disregarded if the couple are not married

c staff may be delighted

$\mathrm{d}$ any best interests decision in relation to the termination of pregnancy must make the interests of the unborn child paramount

e staff should have regard to Article 12 of the European Convention on Human Rights.
5 About pornography:

a it is acceptable for staff to use it for sex education that forms part of the patient's or service user's care plan and has been agreed by the care team

b it refers to magazines, posters, films, DVDs and videos but not books

C for the purposes of policies, no distinction should be made between films classified '18' by the British Board of Film Classification and films classified 'R18'

d assisting a patient or service user to obtain it could be illegal under s.53A of the Sexual Offences Act 2003

e sharing it with other patients should be discouraged. 